\title{
DNA-Templated formation and luminescence of diphenylacetylene dimeric and trimeric complexes
}

\author{
Robert L. Letsinger, * Taifeng Wu, $\dagger$ Jye-Shane Yang $\ddagger$ and Frederick D. Lewis* \\ Received 1st April 2008, Accepted 20th May 2008 \\ First published as an Advance Article on the web 9th June 2008 \\ DOI: $10.1039 / \mathrm{b805452e}$
}

We report on spectral features for two and three diphenylacetylene chromophores aligned in close proximity in aqueous solution by self assembly of attached oligonucleotide arms. Two duplex systems were examined in detail. One was formed by hybridization (Watson-Crick base pairing) of two oligonucleotide 10-mers, each containing the diphenylacetylene insert. The other was generated by self-folding of a 36-mer oligonucleotide containing two diphenylacetylene inserts. The triplex system was obtained by hybridization (Hoogsteen base pairing) of a 16-mer oligonucleotide diphenylacetylene conjugate to the folded 36-mer hairpin. Formation of duplex and triplex entities from these conjugates was demonstrated experimentally by thermal dissociation and spectroscopic studies. The UV and CD spectra for the duplex systems exhibit bands in the $300-350 \mathrm{~nm}$ region attributable to exciton coupling between the two chromophores, and the emission spectra show a strong band centered at $410 \mathrm{~nm}$ assigned to excimer fluorescence. Addition of the third strand to the hairpin duplex has little effect on the CD spectrum in the $300-350 \mathrm{~nm}$ region, but leads to a negative band at short wavelengths characteristic of a triplex and to a strongly enhanced band at $410 \mathrm{~nm}$ in the fluorescence spectrum. The third strand alone shows a broad fluorescence band at $\sim 345-365 \mathrm{~nm}$, but this band is virtually absent in the triplex system. A model for the triplex system is proposed in which two of the three aligned diphenylacetylenes function as a ground state dimer that on excitation gives rise to the exciton coupling observed in the UV and CD spectra and to the excimer emission observed in the fluorescence spectrum. Excitation of the third chromophore results in enhanced excimer fluorescence, as a consequence of energy transfer from the locally excited singlet of one chromophore to the ground state dimer formed by the other two chromophores.

\section{Introduction}

Hybridization of oligonucleotides bearing organic fragments provides a convenient means to orient those organic moieties in dilute aqueous solution. ${ }^{1,2}$ We have previously exploited construct I (Chart 1), where oligo ${ }^{\mathrm{i}}$ is complementary to oligo ${ }^{\mathrm{ii}}$ and oligo $\mathrm{oii}^{\mathrm{iii}}$ is complementary to oligo ${ }^{\mathrm{iv}}$, to study the photophysical and photochemical properties of two stilbenedicarboxamide or perylenediimide chromophores aligned in close proximity. ${ }^{3-6}$ A similar system served to position two steroid units to generate a hydrophobic pocket in an aqueous medium. ${ }^{7}$ For the stilbenedicarboxamide

\section{Construct I}

Construct I I

$$
\begin{aligned}
& \text { 5' oligo - organic fragment - oligo iii } 3 \text { ' } \\
& 3 \text { ' oligo }{ }^{i i} \text { - organic fragment - oligo }{ }^{\text {iv }} 5 \text {, } \\
& 5^{\prime} \text { oligo - organic fragment - oligo ii } 3 \text { ' } \\
& 3^{\prime} \text { oligo }{ }^{i i i} \text { - organic fragment - oligo }{ }^{\text {iv }} 5 \text { ' } \\
& 3 \text { ' oligo }{ }^{\text {iv }} \text { - organic fragment }- \text { oligo }^{\vee} 5 \text {, }
\end{aligned}
$$

Chart 1 Structures of constructs I and II. case, we found that hybridization resulted in the formation of duplexes in which the two stilbene units form a ground state dimer. ${ }^{4}$ The electronically excited dimer is strongly fluorescent, providing the first example of stilbene excimer fluorescence in fluid solution. DNA templating also enabled us to achieve efficient $[2+$ 2] photodimerization of stilbene units present in solution at very low concentrations.

We report here on properties of two related families of conjugates in which oligonucleotide arms are employed to align carboncarbon triple bonded groups (Chart 2). The linear diphenylacetylene group in the DPA linker in these conjugates absorbs at somewhat higher energy than the stilbene group and cannot undergo cis-trans isomerization on irradiation. Furthermore, $[2+$ 2] photodimerization would be expected to be inefficient since it would lead initially to an anti-aromatic cyclobutadiene. ${ }^{8}$ Conjugates 1-4 are analogues of the conjugates studied in the stilbene system. Along with conjugate $\mathbf{6}$ they serve to probe properties of two adjacent DPA groups in dilute solution. Compounds 5 and $\mathbf{6}$ were designed (a) to explore utility of a new system, construct II (Chart 1), in which oligonucleotide arms could serve to align three DPA chromophores in solution and (b) to investigate excited state properties of DPA groups so aligned. Triplex formation via hybridization of hairpin and single stranded DNA conjugates has been extensively investigated. ${ }^{9,10}$ However, to our knowledge there has been no prior report of the use of triplex formation to align three chromophores.

Department of Chemistry, Northwestern University, Evanston, IL, 60208-

3113.E-mail:rletsinger@ameritech.net,fdl@northwestern.edu

$\dagger$ Present address: 3 Brattle Drive, Apt 5, Arlington MA 02474.

$\$$ Present address: Department of Chemistry, National Taiwan University, Taipei, Taiwan. 


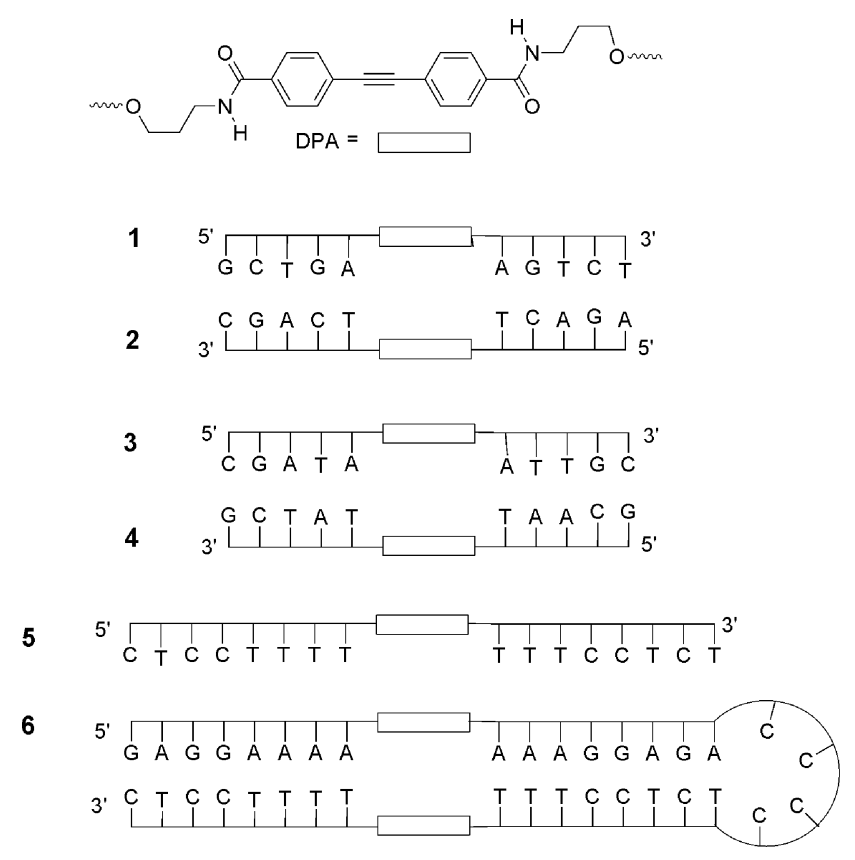

Chart 2 Structures of the DPA linker and conjugates 1-6.

\section{Results and discussion}

\section{Single stranded conjugates 1-5}

Spectral properties of the single stranded conjugates were investigated to provide a base for evaluating the double and triple stranded complexes. The UV absorption spectra of these conjugates show a strong band in the $260 \mathrm{~nm}$ region characteristic of the nucleobases and a weaker band with maxima at 310 and $328 \mathrm{~nm}$ attributed to the diphenylacetylene group, with some overlap from the band for the nucleobases. Their spectra are similar to those for hairpin-forming oligonucleotide-DPA conjugates. ${ }^{11,12}$ The CD spectra of conjugates 3 and 4 (Fig. 1) have minima and maxima near 252 and $275 \mathrm{~nm}$, respectively, characteristic of single stranded oligonucleotides, ${ }^{13}$ and no CD band at wavelengths longer than $300 \mathrm{~nm}$. Similar CD spectra are observed for the other single stranded conjugates.

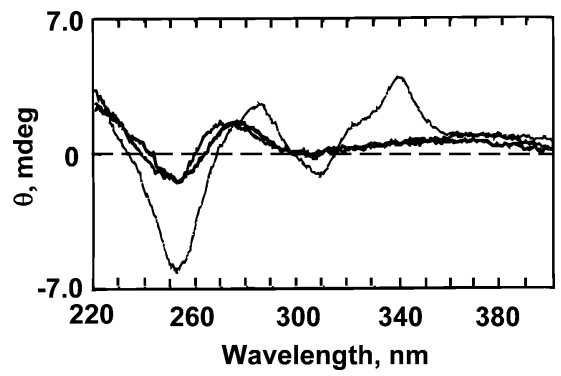

Fig. 1 CD spectra of equimolar amounts $\left(c a .2 \times 10^{-6} \mathrm{M}\right)$ of 3 and 4 (heavy traces) and duplex 3:4 (light trace) in $0.1 \mathrm{M} \mathrm{NaCl}$ at $\mathrm{pH}$ 7.0.

The diol derivative of the DPA linker (Chart 2) is strongly fluorescent in methanol solution, having a band maximum at $351 \mathrm{~nm}$, a quantum yield $\left(\Phi_{\mathrm{fl}}\right)$ of 0.33 , and a fluorescence decay time of 0.60 ns. ${ }^{12}$ The single stranded conjugates $\mathbf{1 - 5}$ also have emission maxima near $350 \mathrm{~nm}$ in aqueous buffer (Fig. 2). Values of $\Phi_{\mathrm{fl}}$ for $\mathbf{1 - 4}$ are $\leq 0.005$ (Table 1) whereas the value for $\mathbf{5}$ is 0.14

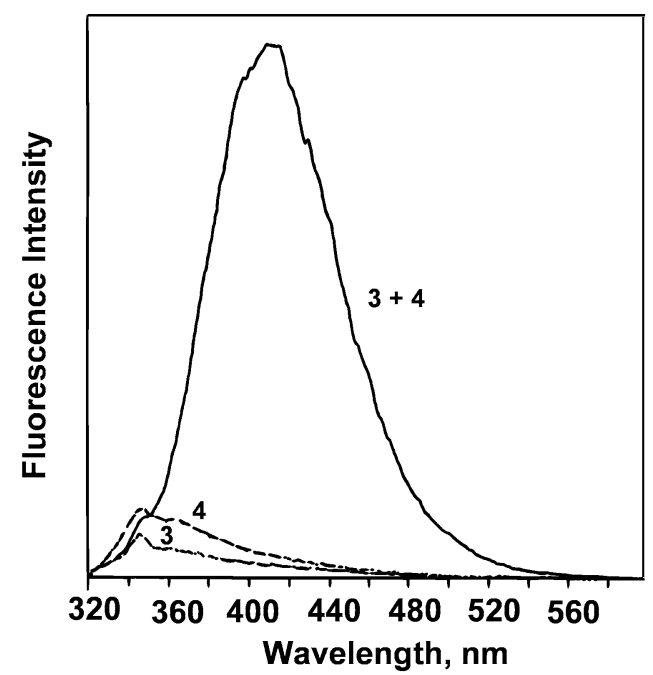

Fig. 2 Fluorescence spectra of conjugates 3 and $\mathbf{4}$ and duplex 3:4 for $c a$. $2 \times 10^{-6} \mathrm{M}$ conjugate in $0.1 \mathrm{M} \mathrm{NaCl}, \mathrm{pH} 7.0$ (Tris- $\mathrm{HCl}$ ) at $0{ }^{\circ} \mathrm{C}$.

Table $1 T_{\mathrm{m}}$ values and fluorescence quantum yields for conjugates in the duplex system (1-4) ${ }^{a}$

\begin{tabular}{lcc}
\hline Conjugates & $T_{\mathrm{m}} /{ }^{\circ} \mathrm{C}$ & $\Phi_{\mathrm{fl}}{ }^{b}$ \\
\hline $\mathbf{1}$ & & $<0.005$ \\
$\mathbf{2}$ & & $<0.005$ \\
$\mathbf{3}$ & & $<0.005$ \\
$\mathbf{4}$ & 36 & $<0.005$ \\
$\mathbf{1 : 2}$ & 34 & 0.01 \\
$\mathbf{3 : 4}$ & & 0.20
\end{tabular}

${ }^{a}$ Experiments were carried out in aqueous $0.1 \mathrm{M} \mathrm{NaCl}$ at $\mathrm{pH} 7.0$ (Tris$\mathrm{HCl}$ ) containing $\sim 5 \mu \mathrm{M}$ of each oligomer. ${ }^{b}$ Fluorescence quantum yields were determined using $315 \mathrm{~nm}$ excitation of nitrogen-purged solutions at $20^{\circ} \mathrm{C}$.

Table $2 T_{\mathrm{m}}$ values, fluorescence quantum yields, and lifetime data for conjugates in the triplex system $(\mathbf{5 , 6})^{b}$

\begin{tabular}{llll}
\hline Conjugate & $T_{\mathrm{m}} /{ }^{\circ} \mathrm{C}$ & $\Phi_{\mathrm{fl}}{ }^{b}$ & $\tau / \mathrm{ns}^{c}$ \\
\hline $\mathbf{5}$ & & 0.14 & 0.5 \\
$\mathbf{6}$ & $>75$ & 0.29 & $2.7(83), 8.0(17)$ \\
& $\mathbf{5 : 6}$ & & $4.0(80), 10.1(20)^{d}$ \\
& $18,>75$ & 0.43 & $2.6(69), 9.6(31)$ \\
& & & $2.7(88), 12.5(12)^{d}$
\end{tabular}

${ }^{a}$ Experiments were carried out in aqueous $1.0 \mathrm{NaCl}$ at $\mathrm{pH} 7.0$ (Tris- $\mathrm{HCl}$ ) containing $\sim 5 \mu \mathrm{M}$ of each oligomer. ${ }^{b}$ Quantum yields determined at $20^{\circ} \mathrm{C}$ using $315 \mathrm{~nm}$ excitation. ${ }^{c}$ Fluorescence decays determined using $315 \mathrm{~nm}$ excitation at $360 \mathrm{~nm}$ for single stranded conjugate 5 and at $440 \mathrm{~nm}$ emission for 6 and 5:6. Values in parentheses are percentage of total decay. Values determined at $20^{\circ} \mathrm{C}$ except as noted. ${ }^{d}$ Values determined at $5{ }^{\circ} \mathrm{C}$.

(Table 2). The higher value for $\mathbf{5} v s . \mathbf{1 - 4}$ is attributed to the absence of purine bases in the former conjugate. The fluorescence of DPA in hairpin-forming conjugates is strongly quenched by purine bases, which have lower oxidation potentials than the pyrimidine bases. ${ }^{12}$ Fluorescence quenching occurs via an electron transfer process in which singlet DPA is reduced by an adjacent purine. The fluorescence decay of $\mathbf{5}$ can be fit as a single exponential with a decay time of $0.5 \mathrm{~ns}$ (Table 2), similar to that of the DPA diol in methanol. 


\section{Duplex system}

Hybridization of conjugates $\mathbf{1}$ and $\mathbf{2}$ or $\mathbf{3}$ and $\mathbf{4}$ (Chart 2) results in the formation of duplex structures. The melting curve for the duplex 1:2 provides values of $T_{\mathrm{m}}\left(36^{\circ} \mathrm{C}\right)$ and hypochromicity (0.13) similar to those for the duplex formed by the analogous stilbene conjugates., ${ }^{3,4}$ The slightly lower value of $T_{\mathrm{m}}$ for duplex 3:4 reflects the replacement of one of the $\mathrm{G}-\mathrm{C}$ base pairs in 1:2 with a weaker A-T base pair in 3:4. The duplex UV absorption spectra have the same band maxima as those for the single stranded conjugates; however the ratio of $310 / 328 \mathrm{~nm}$ band intensities is larger for the duplex than for the single strand conjugates (1.35 vs. 1.10). The increase in band ratios without a shift in the absorption maxima is similar to that observed for perylenediimide dimers $^{6,14}$ and is attributed to exciton coupling between the two DPA chromophores in the duplex.

The CD spectrum for duplex 3:4 displays a minimum at $252 \mathrm{~nm}$ and maximum at $285 \mathrm{~nm}$ (Fig. 1), as expected for a duplex containing both A:T and G:C base pairs. ${ }^{13}$ In addition it displays a minimum at $308 \mathrm{~nm}$ and a maximum at $340 \mathrm{~nm}$, a spectral region where the DPA insert absorbs and absorption by bases is very weak. Similar bisignate $C D$ bands have been observed for the duplex conjugates possessing stilbene ${ }^{5}$ or perylenediimide ${ }^{6}$ chromophores and are assigned to exciton coupling between the two chromophores. The relative intensity of the long wavelength (DPA) vs. shorter wavelength (nucleobase) CD bands indicates that the two chromophores are in close proximity and do not have perfectly aligned long axes, which would result in zero rotational strength. ${ }^{6,13}$

The fluorescence spectra of duplexes 1:2 and 3:4 (Fig. 2) are redshifted with respect to those of the single stranded conjugates. The appearance of red-shifted emission is similar to the fluorescence of the analogous stilbene-containing duplexes ${ }^{3,4}$ and is attributed to the singlet excited DPA dimer or excimer. To our knowledge excimer fluorescence from unsubstituted diphenylacetylene or its derivatives has not been previously observed in fluid solution. However, Brocklehurst et al. ${ }^{15}$ have assigned an emission having a maximum at $390 \mathrm{~nm}$ observed upon warming a squalene glass containing diphenylacetylene following $\gamma$-irradiation to a diphenylacetylene excimer.

Both 1:2 and 3:4 display excimer fluorescence but little or no monomer fluorescence. However, the quantum yield for fluorescence is significantly larger for 3:4 than for 1:2 (Table 1). The relatively strong excimer fluorescence for 3:4 indicates that the excimer is quenched less efficiently than the monomer by an adjacent A-T base pair. This observation is consistent with the lower singlet energy and hence the lower driving force for photoinduced electron transfer quenching of the singlet excited excimer vs. the monomer. ${ }^{16}$ The lower value of $\Phi_{\mathrm{fl}}$ for the 1:2 vs. 3:4 excimer is attributed to the location of a $\mathrm{G}-\mathrm{C}$ base pair closer to the DPA chromophores in 1:2. Guanine has a lower oxidation potential than adenine and is thus more readily oxidized. ${ }^{17}$ Quenching of singlet stilbene fluorescence in hairpinforming conjugates having a single $\mathrm{G}-\mathrm{C}$ base pair is strongly dependent upon the location of guanine. ${ }^{18}$

Molecular modeling of duplex systems having aligned perylenediimide $^{6}$ or pyrenediamide ${ }^{19}$ linkers indicates that the two chromophores are buried between the two duplex base pair domains, a geometry which minimizes exposure of their hydrophobic $\pi$-surfaces to water and allows $\pi$-stacking between the chromophores and with the adjacent base pair. We propose that duplexes 1:2 and 3:4 adopt similar geometries having buried DPA chromophores, as shown schematically in Chart 3, rather than an exposed geometry having aligned DPA long axes, as suggested by the linear structures in Chart 2. The proposed duplex geometry permits orbital overlap between DPA chromophores and is consistent with the observation of exciton coupled UV and CD spectra and excimer fluorescence.
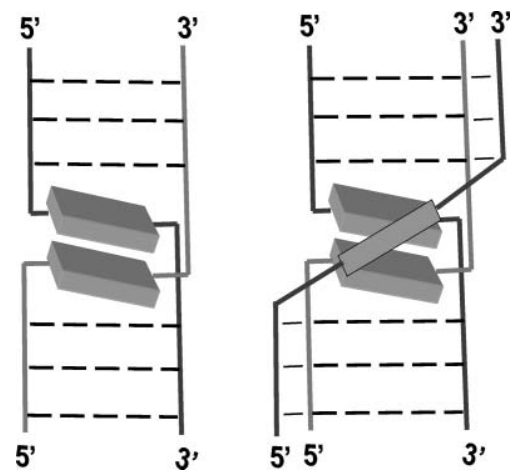

Chart 3 Proposed structures for duplex (left) and triplex (right) conjugates.

The DPA duplex conjugates were irradiated using the same experimental conditions previously employed with the stilbene oligonucleotide duplex. ${ }^{3,20}$ Photobleaching was inefficient relative to the stilbene conjugates, for which photobleaching resulting from $[2+2]$ photodimerization was complete in 10 minutes. The complex product mixtures obtained from 1:2 or 3:4 have not been characterized.

\section{Triplex system}

Conjugates 5 and 6 (Chart 2) were designed to explore the interactions of one, two, or three DPA fragments. In principle, an oligonucleotide with sequence $\mathbf{6}$ can align DPA groups either by hybridization of two strands to form an extended duplex or by folding to give a hairpin structure. We represent the structure as a hairpin (Chart 2) since extensive work with oligonucleotides comprising a short segment, such as -CCCC-, flanked by complementary oligonucleotide arms has shown that the hairpin structure is preferred. ${ }^{21,22}$ Such structures are distinguished by their high thermal stability. Conjugate 5 was designed to bind to conjugate 6 via Hoogsteen $\mathrm{A}: \mathrm{T}$ and $\mathrm{G}: \mathrm{C}$ base pairing ${ }^{23}$ thereby bringing three DPA groups into proximity.

Conjugate 6 indeed self-hybridized to give an unusually stable structure, as shown by a $T_{\mathrm{m}}$ value for thermal dissociation $>75^{\circ} \mathrm{C}$ in $1.0 \mathrm{M} \mathrm{NaCl}$ at pH 7.0 (Fig. 3a). The UV, CD (Fig. 4), and fluorescence spectra of 6 (Fig. 5) mirror those for the duplex 3:4. The long-wavelength region of the $\mathrm{CD}$ spectrum displays a minimum at $308 \mathrm{~nm}$ and maximum at $337 \mathrm{~nm}$, similar to those for the duplex (Fig. 1). A strong excimer band centered near $410 \mathrm{~nm}$ dominates the fluorescence spectrum. The fluorescence decay of hairpin 6 is biexponential (Table 2), the major and minor components having decay times of $4.0 \mathrm{~ns}$ and $10.1 \mathrm{~ns}$ respectively at $5{ }^{\circ} \mathrm{C}$. The dual-exponential character of the excimer fluorescence is analogous to the behavior of DNA-templated stilbene 


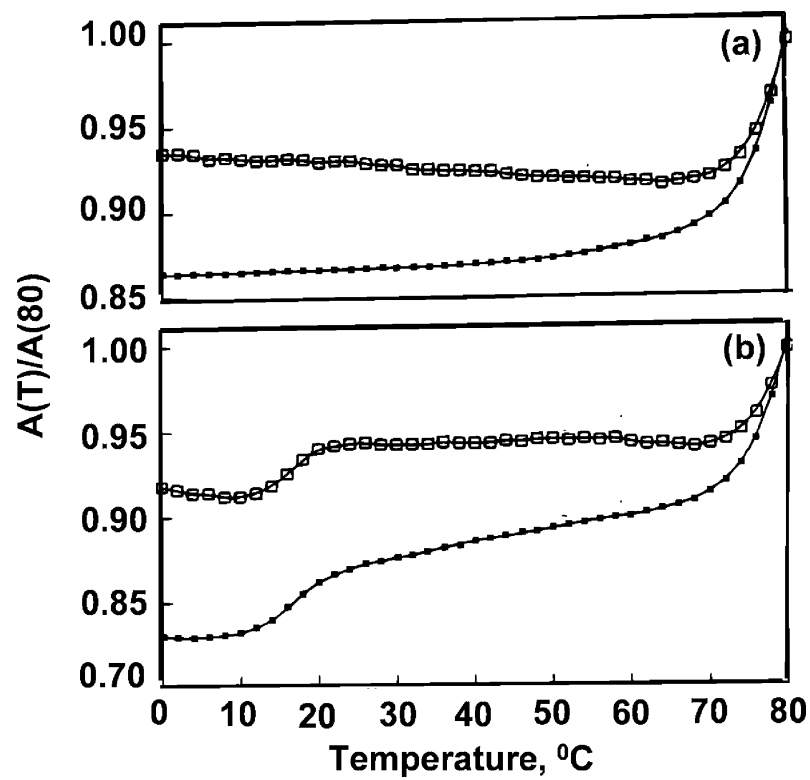

Fig. 3 Thermal dissociation curves in $1.0 \mathrm{M} \mathrm{NaCl}, \mathrm{pH} 7.0$ for (a) hairpin 6 and (b) 6 plus an equimolar amount of 5 , measured at $325 \mathrm{~nm}(\square)$ and at $260 \mathrm{~nm}(\boldsymbol{\square})$.

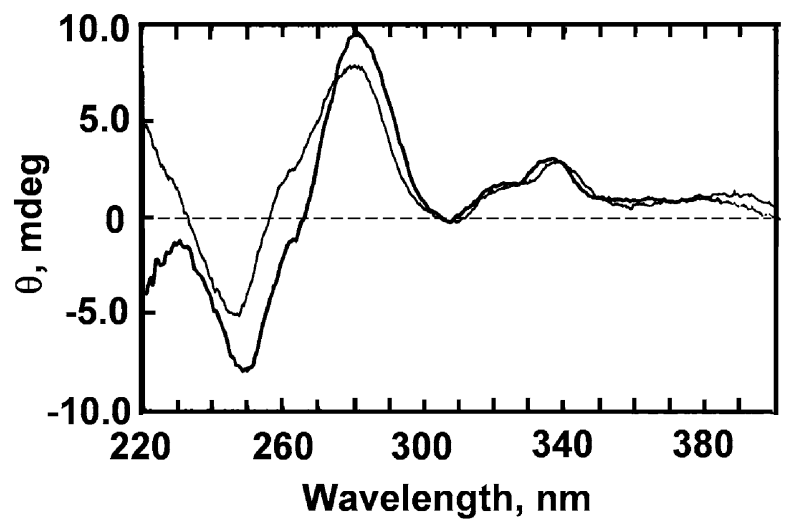

Fig. 4 CD spectra of equimolar amounts $\left(c a .2 \times 10^{-6} \mathrm{M}\right)$ of 6 (light trace) and 6 plus 5 (dark trace) in $1.0 \mathrm{M} \mathrm{NaCl}$ at $\mathrm{pH} 5.5$ (phosphate buffer).

excimers, which was attributed to multiple duplex geometries. ${ }^{4}$ Both decay times are significantly longer than those of either DPA diol or single stranded conjugate $\mathbf{5}$. The increase in lifetime for excimer $v s$. monomer fluorescence is typical of arene excimers and is a consequence of less favorable Franck-Condon factors for emission of the excimer. ${ }^{24}$

The thermal dissociation profile for a $1: 1$ mixture of hairpin 6 and conjugate 5 displays two transitions (Fig. 3b) in contrast to the single transition for $\mathbf{6}$ alone (Fig. 3a). The higher temperature transition $\left(>75{ }^{\circ} \mathrm{C}\right)$ corresponds to melting of hairpin $\mathbf{6}$, and the lower transition is attributed to conversion of a triplex oligonucleotide structure (5:6) to hairpin $\mathbf{6}$ and free conjugate 5. In view of observations that triple stranded complexes containing cytosines in the third strand are strongly $\mathrm{pH}$ dependen $\mathrm{t}^{25}$ and that protonation of cytosines in the third strand of a triplex enhances triplex stability, ${ }^{26,27}$ we also compared the thermal stability of

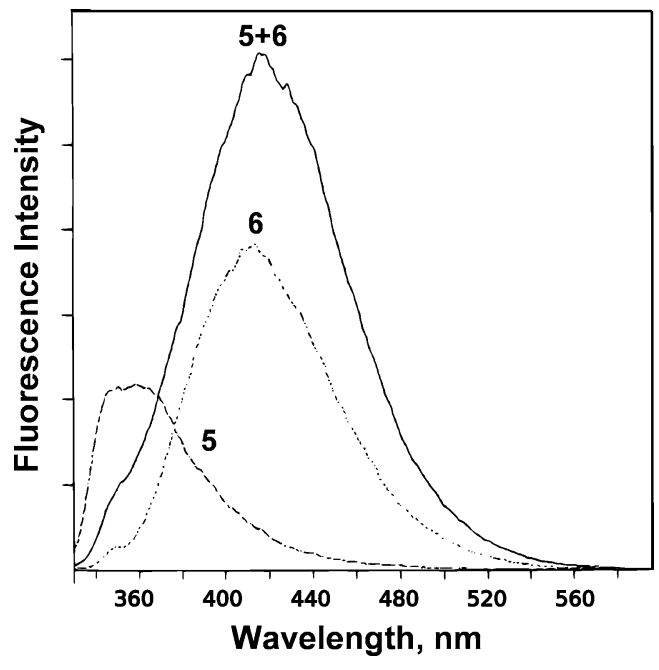

Fig. 5 Fluorescence spectra in $1.0 \mathrm{M} \mathrm{NaCl}$ at $0{ }^{\circ} \mathrm{C}$ for equimolar amounts (ca. $2 \times 10^{-6} \mathrm{M}$ conjugate) of $\mathbf{5}, \mathbf{6}$, and 5 plus $\mathbf{6}$.

triplex structure 5:6 at pH 6.5 and $\mathrm{pH} 5.5$ (Fig. 6). The increase in the $T_{\mathrm{m}}$ value for the first break from $17{ }^{\circ} \mathrm{C}$ to $48{ }^{\circ} \mathrm{C}$ with a $\mathrm{pH}$ change from $\mathrm{pH} 6.5$ to $5.5(0.1 \mathrm{M} \mathrm{NaCl})$ is indicative of the presence of a triple stranded oligonucleotide array for the 5:6 complex at the lower temperatures. The negative band in the short-wavelength region of the CD spectrum $(<230 \mathrm{~nm}$, Fig. 4$)$ is characteristic of a triplex structure, ${ }^{10,13}$ providing further support for triplex formation. At longer wavelengths $(>300 \mathrm{~nm}$ ) the CD spectrum of 5:6 is similar to that of $\mathbf{6}$.

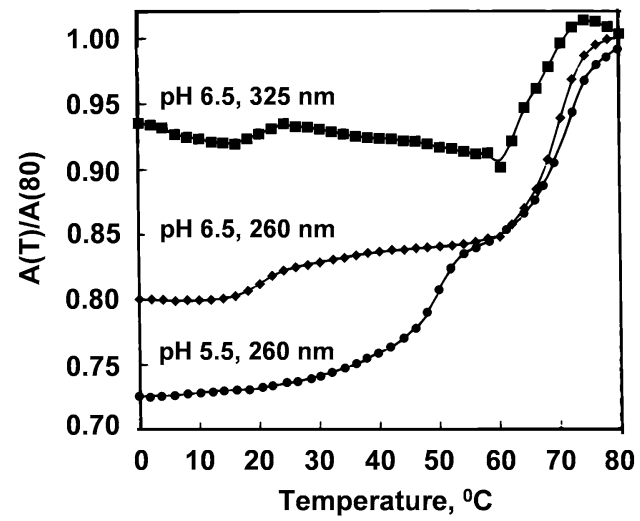

Fig. 6 Thermal dissociation curves for complex 5:6 at pH 5.5 and 6.5.

The fluorescence spectrum for the triplex formed by adding one equivalent of 5 to $\mathbf{6}$ is particularly revealing (Fig. 5). Three features stand out: (a) the band is at approximately the same position ( $\sim 410 \mathrm{~nm})$ as that for the hairpin duplex alone, (b) the intensity is considerably greater than that for the hairpin duplex and (c) monomer fluorescence characteristic of $\mathbf{5}$ is essentially absent. In agreement with the $1: 1$ stoichiometry for the triplex, addition of a second equivalent of $\mathbf{5}$ to the triplex 5:6 results in overlapping monomer and excimer fluorescence. The fluorescence decay time for system 5:6 can be fit as a dual exponential having decay times similar to those for $\mathbf{6}$ (Table 2). Similar decay times are observed at temperatures below or near the triplex $T_{\mathrm{m}}(5$ and $20^{\circ} \mathrm{C}$, respectively). 
The observation of similar exciton coupled CD spectra (Fig. 4) and excimer fluorescence for the duplex 6 and triplex 5:6 (Fig. 5) indicates that the geometry of the two DPA chromophores in the duplex 6 remains largely unaltered upon formation of the triplex. The geometry of the Watson-Crick base pair domains in triplexes having canonical T:AT and $\mathrm{C}^{+}$: $\mathrm{GC}$ triplets is known to be slightly underwound, but otherwise similar to that of B-DNA. ${ }^{23}$ We propose that Hoogsteen base pairing results in location of the DPA chromophore of conjugate 5 near the two DPA chromophores of duplex 6, which remain "buried" between base-pair domains (Chart 3). This geometric arrangement for the three DPA chromophores can account for the absence of monomer fluorescence from 5 as well as the similar CD and fluorescence spectra for the hairpin duplex 6 and the triplex 5:6. The proximity of the three DPA chromophores in 5:6 should permit efficient energy transfer from the higher energy monomer in $\mathbf{5}$ to the ground state dimer in $6 .{ }^{16}$ Assuming similar molar absorbance values for the three DPA chromophores, a $c a$. 50\% increase in excimer fluorescence intensity would be expected upon addition of 5, as is observed (Fig. 5). Alternative triplex structural models can be envisioned; however the one presented in Chart 3 has the merits of simplicity and accommodation of the available spectral data.

Irradiation of 5:6 under the conditions used for 3:4 likewise led to relatively slow bleaching of the UV absorption bands due to the DPA groups. Ion exchange reversed phase chromatography of the products from 10 min irradiation showed the presence of unreacted starting oligomers (5 and $\mathbf{6}$ ) and a small band with four peaks eluting after $\mathbf{5}$ and $\mathbf{6}$, indicating formation of larger oligomers. The products were not further investigated.

\section{Summary}

We have utilized construct I to examine excited state properties of two aligned organic inserts containing carbon-carbon triple bonds (DPA groups). CD and fluorescence data for aqueous solutions of duplex 3:4 and hairpin 6 reveal that chromophores so organized in dilute solution interact both in the ground state and upon photoexcitation. Efficient quenching of fluorescence occurs in single stranded DPA conjugates possessing adenine bases near the chromophore, but not in the duplexes having only A:T pairs near the chromophores. Guanine, a more effective quencher than adenine, significantly reduces excimer fluorescence even when in the second nucleotide position to the DPA chromophore in a duplex, as shown by the relative fluorescence intensities of duplexes 1:2 and 3:4.

Data provided by melting curves, fluorescence spectra, quantum yields, lifetime measurements, and CD spectra provide convincing evidence for the utility of construct II in aligning three organic entities in proximity in solution. The triplex system 5:6 displays fluorescence with the same band shape and decay times as found for the corresponding duplex 6, but with greater fluorescence intensity. We conclude from the fluorescence data that all three chromophores participate in the excitation step leading to fluorescence, but emission stems from an excimer formed by only two chromophores. Delocalization to form a fluorescent complex comprising three chromophores therefore appears unfavorable even in the constrained system provided by construct II. In contrast to the fluorescence data, intensities of the CD bands were not enhanced when the third strand was hybridized to the duplex. We suggest as a plausible model to rationalize the CD and fluorescence data that the two DPA groups of the hairpin 6 function as a ground state dimer which on excitation gives rise to the exciton coupling observed by $\mathrm{CD}$ and excimer emission observed by fluorescence, and that excitation of the third DPA group leads to enhancement of the excimer fluorescence band by means of energy transfer to the lower energy excimer excited state. Thus the third strand provides a light-harvesting chromophore for enhanced excimer fluorescence.

\section{Experimental}

\section{Materials}

Conjugates 1-6 were prepared using conventional nucleoside phosphoramidite reagents and DPA-diol modified by DMT at one end and an $N, N$-di-isopropylphosphoamidite group at the other, following the procedure used is preparing related stilbene oligonucleotide conjugate ${ }^{3,4}$ and adapted to synthesis of hairpin structures consisting of complementary oligonucleotides joined by a DPA bridge. ${ }^{12}$ The preparation, purification, and characterization of the conjugates followed the method of Letsinger and $\mathrm{Wu}^{20}$ as implemented by Lewis and coworkers. ${ }^{28}$ The oligomers were first purified as DMT-on derivative by RP HPLC; then after removal of the DMT group by treatment with $80 \%$ acetic acid, the conjugates were again subjected to RP HPLC. Subsequent IE HPLC showed a single peak in each case.

\section{Methods}

Thermal dissociation curves were obtained for aqueous $\mathrm{NaCl}$ solutions $5 \times 10^{-6} \mathrm{M}$ in each oligomers using a Perkin Elmer Lambda 2 UV spectrophotometer equipped with a temperature programmer for automatically increasing the temperature at the rate of $0.8{ }^{\circ} \mathrm{C} \mathrm{min}^{-1}$. CD spectra were recorded using a Jasco J500 $\mathrm{C}$ spectropolarimeter. Fluorescence spectra were determined using a Perkin Elmer LS 50B spectrofluorimeter equipped with a RM6 Lauda circulating bath for controlling the temperature. Fluorescence quantum yields, measured using DPA diol in methanol as a reference, ${ }^{12}$ were determined for deoxygenated aqueous solutions containing $\sim 5 \times 10^{-6} \mathrm{M}$ conjugate in Tris- $\mathrm{HCl}$ buffer at $\mathrm{pH} 7.0$, as described previously. ${ }^{20}$ Fluorescence decay times were measured using a PTI-LS1 single photo counting apparatus. Fluorescence decays were analyzed using the method of James et al. ${ }^{29}$

\section{Acknowledgements}

We thank Mahesh Hariharan for assistance in preparation of the manuscript. This research was supported by grants from the National Institute of General Medical Sciences to RLL (GM 10265) and from the National Science Foundation to FDL (CHE9301381).

\section{References}

1 X. Li and D. R. Liu, DNA-Templated organic synthesis: Nature's strategy for controlling chemical reactivity applied to synthetic molecules, Angew. Chem., Int. Ed., 2004, 43, 4848-4870. 
2 J. Svoboda and B. König, Templated photochemistry: Toward catalysts enhancing the efficiency and selectivity of photoreactions in homogeneous solutions, Chem. Rev., 2006, 106, 5413-5430.

3 R. L. Letsinger and T. Wu, Control of excimer emission and photochemistry of stilbene units by oligonucleotide hybridization, J. Am. Chem. Soc., 1994, 116, 811-812.

4 F. D. Lewis, T. Wu, E. L. Burch, D. M. Bassani, J.-S. Yang, S. Schneider, W. Jäger and R. L. Letsinger, Hybrid oligonucleotides containing stilbene units. Excimer fluorescence and photodimerization, J. Am. Chem. Soc., 1995, 117, 8785-92.

5 R. L. Letsinger, T. F. Wu and R. Elghanian, Chemical and photochemical ligation of oligonucleotide blocks, Nucleosides \& Nucleotides, 1997, 16, 643-652.

6 Y. Zheng, H. Long, G. C. Schatz and F. D. Lewis, Duplex and hairpin dimer structures for perylene diimide-oligonucleotide conjugates, Chem. Commun., 2005, 4795-4797.

7 R. L. Letsinger and S. Chaturvedi, Tailored hydrophobic cavities in oligonucleotide-steroid conjugates, Bioconjugate Chem., 1998, 9, 826830 .

8 G. Büchi, C. W. Perry and E. W. Robb, Photochemical reactions. XI. Diphenylacetylene, J. Org. Chem., 1962, 27, 4106-4107.

9 S. Bevers, S. Schutte and L. W. Mclaughlin, Naphthalene and perylene based linkers for the stabilization of hairpin triplex, J. Am. Chem. Soc., 2000, 122, 5905-5919.

10 I. Trkulja and R. Häner, Monomeric and heterodimeric triple helical DNA mimics, J. Am. Chem. Soc., 2007, 129, 7982-7989.

11 F. D. Lewis, X. Liu, S. E. Miller and M. R. Wasielewski, Electronic interactions between $\pi$-stacked DNA base pairs and diphenylacetylene4,4'-dicarboxamide in hairpin DNA, J. Am. Chem. Soc., 1999, 121, 9746-9747.

12 F. D. Lewis, X. Liu, S. E. Miller, R. T. Hayes and M. R. Wasielewski, Formation and decay of localized contact radical ion pairs in DNA hairpins, J. Am. Chem. Soc., 2002, 124, 1402014026.

13 N. Berova, K. Nakanishi and R. W. Woody, Circular Dichroism, WileyVCH, New York, 2000.

14 A. E. Clark, C. Qin and A. D. Q. Li, Beyond exciton theory: A time-dependent DFT and Franck-Condon study of perylene diimide and its chromophoric dimer, J. Am. Chem. Soc., 2007, 129, 75867595.

15 B. Brocklehurst, D. C. Bull, M. Evans, P. M. Scott and G. Stanney, Excimer fluorescence of trans-stilbene and diphenylacetylene, J. Am. Chem. Soc., 1975, 97, 2977-2978.
16 F. D. Lewis, Y. Zhang and R. L. Letsinger, Bispyrenyl excimer fluorescence: A sensitive oligonucleotide probe, J. Am. Chem. Soc., 1997, 119, 5451-5452.

17 C. A. M. Seidel, A. Schulz and M. H. M. Sauer, Nuclobase-specific quenching of fluorescent dyes. 1. Nucleobase one-electron redox potentials and their correlation with static and dynamic quenching efficiencies, J. Phys. Chem., 1996, 100, 5541-5553.

18 F. D. Lewis, T. Wu, X. Liu, R. L. Letsinger, S. R. Greenfield, S. E. Miller and M. R. Wasielewski, Dynamics of photoinduced charge separation and charge recombination in synthetic DNA hairpins with stilbenedicarboxamide linkers, J. Am. Chem. Soc., 2000, 122, 28892902.

19 S. M. Langenegger and R. Häner, Excimer formation by interstrand stacked pyrenes, Chem. Commun., 2004, 2792-2793.

$20 \mathrm{R}$. L. Letsinger and T. Wu, Use of a stilbenedicarbxoamide bridge in stabilizing, monitoring, and photochemically altering folded conformations of oligonucleotides, J. Am. Chem. Soc., 1995, 117, 7323-7328.

21 L. E. Xodo, G. Manzini, F. Quadrifoglio, G. Vandermarel and J. Vanboom, DNA hairpin loops in solution-correlation between primary structure, thermostability and reactivity with single-strandspecific nuclease from mung bean, Nucleic Acids Res., 1991, 19, 15051511.

22 J. SantaLucia, R. Kierzek and D. H. Turner, Context dependence of hydrogen-bond free-energy revealed by substitutions in an RNA hairpin, Science, 1992, 256, 217-219.

23 E. Wang and J. Feigon, Structures of nucleic acid triplexes, in Nucleic Acid Structure, ed. S. Neidle, Oxford, UK, 1999.

24 J. B. Birks, Photophysics of Aromatic Molecules, Wiley-Interscience, London, 1970.

25 G. E. Plum and K. J. Breslauer, Thermodynamics of an intramolecular DNA triple-helix - a calorimetric and spectroscopic study of the $\mathrm{pH}$ and salt dependence of thermally-induced structural transitions, J. Mol. Biol., 1995, 248, 679-695.

26 G. E. Plum, Thermodynamics of oligonucleotide triple helices, Biopolymers, 1997, 44, 241-256.

27 R. W. Roberts and D. M. Crothers, Prediction of the stability of DNA triplexes, Proc. Natl. Acad. Sci. USA, 1996, 93, 4320-4325.

28 F. D. Lewis, Y. Wu and X. Liu, Synthesis, structure, and photochemistry of exceptionally stable synthetic DNA hairpins with stilbene diether linkers, J. Am. Chem. Soc., 2002, 124, 12165-12173.

29 D. R. James, A. Siemiarczuk and W. R. Ware, Stroboscopic optical boxcar technique for the determination of fluorescence lifetimes, Rev. Sci. Instrum., 1992, 63, 1710-1716. 